\title{
CHARACTERISTIC FUNCTIONS AND PRODUCTS OF BOUNDED DERIVATIVES
}

\author{
ALEKSANDER MALISZEWSKI
}

(Communicated by Andrew M. Bruckner)

\begin{abstract}
This article is dedicated to the answer to the following question: "Which characteristic functions can be expressed as the product of two or more bounded derivatives?".
\end{abstract}

The real line $(-\infty, \infty)$ is denoted by $\mathbb{R}$, the set of integers by $\mathbb{Z}$ and the set of positive integers by $\mathbb{N}$. The only measure used is Lebesgue measure in $\mathbb{R}$ and each integral should be interpreted as the corresponding Lebesgue integral. The distance between two nonempty subsets $A, B$ of $\mathbb{R}$ will be denoted by $\varrho(A, B)$ (i.e. $\varrho(A, B)=\inf \{|x-y|: x \in A, y \in B\})$. For each set $A \subset \mathbb{R}$, let int $A$ denote its (Euclidean) interior, cl $A$ its closure, fr $A$ its boundary, $|A|$ its outer measure and $\chi_{A}$ its characteristic function.

All functions will be real functions of a real variable. If $I$ is an interval (throughout this paper we deal only with nondegenerate intervals), then $\mathscr{D}(I)$ denotes the family of all derivatives defined on $I$ (in the case of an endpoint of $I$ that belongs to $I$ we consider the corresponding one-sided condition). Let $\mathscr{D}$ denote $\mathscr{D}(\mathbb{R})$.

The terms " $d$-closed", " $d$-interior" ( $d$-int), etc., refer to the Denjoy topology (density topology) on $\mathbb{R}$. (See, e.g., [2], [5].) We say that a function is approximately continuous if and only if it is continuous relative to the Denjoy topology. It is well known that approximately continuous functions are Baire one functions and bounded approximately continuous functions are derivatives (cf., e.g., [1]). We will use an old lemma of Zahorski.

Lemma 1. Let $A$ and $B$ be disjoint, $d$-closed, $G_{\delta}$ sets. Then there is an approximately continuous function $g: \mathbb{R} \rightarrow \mathbb{R}$ such that $0 \leq g \leq 1$ on $\mathbb{R}, g=1$ on $A$ and $g=0$ on $B$ [6, Lemma 12].

For each set $T \in \mathbb{R}$ and each interval $I$, let $\varphi_{T}(I)$ denote the measure of the greatest interval $J$ contained in $I \backslash T$, if any such interval exists, and 0 otherwise (cf., e.g., [4]). If $a$ and $b$ are endpoints of $I$, then we will write also $\varphi_{T}(a, b)$ instead of $\varphi_{T}(I)$.

Received by the editors October 31, 1992 and, in revised form, August 10, 1993 and November 18, 1993; presented at the Summer School on Real Functions Theory, Dubnik, Slovakia, May 1992. 1991 Mathematics Subject Classification. Primary 26A24.

Supported by a KBN Research Grant 2114491 01, 1992-94. 
We say that the set $T \subset \mathbb{R}$ is nonporous at $x$ iff

$$
\lim _{t \rightarrow 0^{+}} \frac{\varphi_{T}(x-t, x+t)}{2 t}=0 .
$$

Also, $T$ is called nonporous if it is nonporous at each of its points. We say that the set $T \subset \mathbb{R}$ is ambiguous iff it is both an $F_{\sigma}$ and a $G_{\delta}$ set.

For the remainder of this article, let $T$ denote a fixed ambiguous and nonporous subset of $\mathbb{R}$, and $S=\mathbb{R} \backslash T$. We will write $\varphi(I)$ instead of $\varphi_{T}(I)$.

For each bounded interval $I$ and $\varepsilon \in(0,1]$, we will denote by $\mathscr{P}(I, \varepsilon)$ the family of all pairs of derivatives $\left(f_{1}, f_{2}\right)$ defined on $\mathrm{cl} I$ which satisfy the following conditions:

(a) $f_{1} \cdot f_{2}=0$ on $T \cap I$,

(b) $f_{1}=f_{2}$ and $\left|f_{1}\right|=1$ on $S \cap I$,

(c) if $a$ is an endpoint of $I$, then $f_{1}(a)=f_{2}(a)=\chi_{S}(a)$,

(d) $\left|f_{1}\right|<2,\left|f_{2}\right|<2$,

(e) $\int_{I} f_{1}=\int_{I} f_{2}$,

(f) $\left|\int_{I} f_{1}-\right| I|| \leq \varepsilon \cdot|I|$,

(g) $\left|\left\{x \in I: f_{1}(x)=f_{2}(x)=1\right\}\right| \geq(1-\varepsilon) \cdot|S \cap I|$.

We will denote by $\mathscr{J}$ the family of all bounded intervals $I$ such that $\mathscr{P}(I, \varepsilon) \neq$ $\varnothing$ for each $\varepsilon \in(0,1]$.

The following lemmas are proved in [4].

Lemma 2. Let $H$ be ambiguous and let $A$ be a nonempty $G_{\delta}$ set in $\mathbb{R}$. Then there is an open interval $I$ such that $I \cap A \neq \varnothing$ and that either $I \cap A \subset H$ or $I \cap A \subset \mathbb{R} \backslash H[4$, Lemma 2].

Lemma 3. Let $J$ be an interval such that $J \cap T \neq \varnothing$. Then $J \cap T$ contains an interval [4, Proposition 8].

Denote by $\Omega$ the first uncountable ordinal. We will use the following wellknown theorem (Cantor-Baire stationary principle).

Theorem 4. Let $\left\{F_{\alpha}\right\}_{\alpha<\Omega}$ be a transfinite descending sequence of closed subsets of $\mathbb{R}$. Then there is $a \xi<\Omega$ such that $F_{\alpha}=F_{\xi}$ for $\alpha \geq \xi[3$, Theorem 2,p. 146].

Define a transfinite ascending sequence $\left\{G_{\alpha}\right\}_{\alpha<\Omega}$ of subsets of $\mathbb{R}$ as follows:

(1) $G_{0}=\varnothing$,

(2) $G_{\alpha+1}=\operatorname{int}\left(S \cup G_{\alpha}\right)$, if $\alpha<\Omega$ is even,

(3) $G_{\alpha+1}=\operatorname{int}\left(T \cup G_{\alpha}\right)$, if $\alpha<\Omega$ is odd,

(4) $G_{\alpha}=\bigcup_{\beta<\alpha} G_{\beta}$, if $\alpha<\Omega$ is a limit ordinal.

Then for each $\alpha<\Omega$, the set $G_{\alpha}$ is open. Note that $G_{\alpha} \neq \mathbb{R}$ implies $G_{\alpha} \neq G_{\alpha+2}$ (cf. Lemma 2), so by Theorem 4, there exists a $\xi<\Omega$ such that $G_{\xi}=\mathbb{R}$.

Lemma 5. Whenever $J$ is a bounded open interval and $\varepsilon>0$ there exist $n \in \mathbb{N}$ and $x_{1}, \ldots, x_{n-1} \in T \cap J$ such that $x_{1}<\cdots<x_{n-1}$ and, setting $x_{0}=\inf J$, $x_{n}=\sup J$, we have

$$
x_{i}-x_{i-1} \leq \varphi\left(x_{i-1}, x_{i}\right)+\varepsilon
$$

for $i \in\{1, \ldots, n\}$.

Proof. Since $T \cap J$ is a totally bounded separable space, we can find elements $x_{1}, \ldots, x_{n-1} \in T \cap J$ such that for each $t \in T \cap J$, there is an $i \in\{1, \ldots, n-1\}$ 
such that $\left|t-x_{i}\right|<\varepsilon / 2$. Set $x_{0}$ and $x_{n}$ as above. Then for $i \in\{1, \ldots, n\}$,

$$
x_{i}-x_{i-1}>\varepsilon \Rightarrow\left[x_{i-1}+\varepsilon / 2, x_{i}-\varepsilon / 2\right] \subset S \Rightarrow \varphi\left(x_{i-1}, x_{i}\right) \geq x_{i}-x_{i-1}-\varepsilon \text {. }
$$

Lemma 6. For every $a, b \in T$ with $a<b$, there exists a strictly increasing sequence $\left(x_{z}\right)_{z \in \mathbf{Z}}$ of elements of $T \cap(a, b)$ such that

(1) $\lim _{z \rightarrow-\infty} x_{z}=a, \lim _{z \rightarrow \infty} x_{z}=b$,

(2) for each $z \in \mathbb{Z}$,

$$
x_{z}-x_{z-1} \leq \varphi\left(x_{z-1}, x_{z}\right)+\left[\varrho\left(\{a, b\},\left\{x_{z-1}, x_{z}\right\}\right)\right]^{2} .
$$

Proof. Since $a, b \in T$, there exists a strictly increasing sequence $\left(y_{z}\right)_{z \in Z}$ of elements of $T \cap(a, b)$ with limit points $a$ and $b$ (cf. Lemma 3). For each $z \in$ $\mathbb{Z}$, use Lemma 5 with $J=J_{z}=\left(y_{z-1}, y_{z}\right)$ and $\varepsilon=\left[\varrho\left(\{a, b\},\left\{y_{z-1}, y_{z}\right\}\right)\right]^{2}$, and finds points $x_{z, 1}, \ldots, x_{z, n_{z}-1} \in T \cap J_{z}$ such that $x_{z, 1}<\cdots<x_{z, n_{z}-1}$, and setting $x_{z, 0}=y_{z-1}, x_{z, n_{z}}=y_{z}$, we have for $i \in\left\{1, \ldots, n_{z}\right\}$,

$$
x_{z, i}-x_{z, i-1} \leq \varphi\left(x_{z, i-1}, x_{z, i}\right)+\left[\varrho\left(\{a, b\},\left\{y_{z-1}, y_{z}\right\}\right)\right]^{2} .
$$

Arrange all elements of the set $\left\{x_{z, i}: z \in \mathbb{Z}, i \in\left\{1, \ldots, n_{z}\right\}\right\}$ in a strictly increasing sequence $\left(x_{z}\right)_{z \in \mathbb{Z}}$. It is easy to verify that this sequence possesses desired properties.

Lemma 7. Whenever $D \subset \mathbb{R}$ is measurable and bounded, and $\varepsilon_{1}, \varepsilon_{2} \in(-1,1)$, we can find approximately continuous functions $f_{1}, f_{2}: \mathbb{R} \rightarrow \mathbb{R}$ such that

(1) $f_{1} \cdot f_{2}=0$ on $\mathbb{R}$,

(2) $f_{1}=f_{2}=0$ on $\mathbb{R} \backslash D$,

(3) $\left|f_{1}\right|<2,\left|f_{2}\right|<2$,

(4) $\int_{D} f_{j}=\varepsilon_{j} \cdot|D|(j \in\{1,2\})$.

Proof. If $|D|=0$, then set $f_{1}=f_{2}=0$. Otherwise find disjoint measurable sets $D_{1}, D_{2}$ such that $\left|D_{1}\right|=\left|D_{2}\right|$ and $D_{1} \cup D_{2}=D$. For $j \in\{1,2\}$, first find a closed set $C_{j} \subset d$-int $D_{j}$ such that $\left|C_{j}\right|>\left|\varepsilon_{j}\right| \cdot\left|D_{j}\right|$ and then, using Lemma 1 , find an approximately continuous function $g_{j}: \mathbb{R} \rightarrow \mathbb{R}$ such that $0 \leq g_{j} \leq 1$ on $\mathbb{R}, g_{j}(x)=1$ for $x \in C_{j}$ and $g_{j}(x)=0$ for $x \notin D_{j}$. Finally observe that for $j \in\{1,2\}$,

$$
\left|\frac{\varepsilon_{j} \cdot|D|}{\int_{D_{j}} g_{j}}\right| \leq \frac{2\left|\varepsilon_{j}\right| \cdot\left|D_{j}\right|}{\left|C_{j}\right|}<2
$$

and set

$$
f_{j}=g_{j} \cdot \frac{\varepsilon_{j} \cdot|D|}{\int_{D_{j}} g_{j}} .
$$

It is easy to verify that the requirements of the lemma are fulfilled.

The following two lemmas are due to S. Konjagin. They have never been published.

Lemma 8. Let $r_{1}, \ldots, r_{n} \geq 0$. Then there exist numbers $t_{1}, \ldots, t_{n} \in\{-1,1\}$ such that for $k, l \in\{1, \ldots, n\}, k \leq l$, we have

$$
\left|\sum_{s=k}^{l} t_{s} r_{s}\right| \leq 2 \max \left\{r_{s}: s \in\{k, \ldots, l\}\right\} .
$$


Proof. We will use induction on $n$.

(1) If $n=1$, then we set $t_{1}=1$.

(2) Assume that the assertion holds for some $n \in \mathbb{N}$. Let $r_{1}, \ldots, r_{n+1}$ be nonnegative numbers and choose $t_{1}, \ldots, t_{n} \in\{-1,1\}$ according to the induction assumption. Suppose that both the sequence $\left(t_{1}, \ldots, t_{n}, 1\right)$ and the sequence $\left(t_{1}, \ldots, t_{n},-1\right)$ do not satisfy our requirements. Then there exist $k, l \in\{1, \ldots, n\}$ such that

$$
\sum_{s=k}^{n} t_{s} r_{s}+r_{n+1}>2 \max \left\{r_{s}: s \in\{k, \ldots, n+1\}\right\} \geq 2 r_{n+1}
$$

and

$$
\sum_{s=l}^{n} t_{s} r_{s}-r_{n+1}<-2 \max \left\{r_{s}: s \in\{l, \ldots, n+1\}\right\} \leq-2 r_{n+1} .
$$

We may assume that $k \leq l$ (the opposite case is similar). Subtracting the two above inequalities we get

$$
\sum_{s=k}^{l-1} t_{s} r_{s}+2 r_{n+1}>2 \max \left\{r_{s}: s \in\{k, \ldots, n+1\}\right\}+2 r_{n+1},
$$

contrary to the induction assumption. This completes the proof.

Lemma 9. Given a countable family of nonoverlapping intervals $\mathscr{K}$ and a nonnegative function $r: \mathscr{K} \rightarrow \mathbb{R}$ such that $\sum_{K \in \mathscr{K}} r(K)<\infty$, we can find a function $t: \mathscr{K} \rightarrow\{-1,1\}$ such that for every interval $I \subset \mathbb{R}$,

$$
\left|\sum_{K \subset I, K \in \mathscr{K}} t(K) \cdot r(K)\right| \leq 2 \sup \{r(K): K \subset I, K \in \mathscr{K}\} .
$$

Proof. Let $K_{1}, K_{2}, \ldots$ be a sequence of all elements of $\mathscr{K}$. For each $k \in \mathbb{N}$, apply Lemma 8 and find $t_{k, 1}, \ldots, t_{k, k} \in\{-1,1\}$ such that for every interval $I \subset \mathbb{R}$,

$$
\left|\sum_{K_{n} \subset I, n \leq k} t_{k, n} \cdot r\left(K_{n}\right)\right| \leq 2 \max \left\{r\left(K_{n}\right): K_{n} \subset I, n \leq k\right\} .
$$

In this way we get a triangular matrix $\left[t_{k, n}\right]_{k \in \mathbb{N}, n \leq k}$. Next we proceed by induction. Let $t\left(K_{1}\right)$ be a number which appears in the first column of this matrix infinitely many times. If we have already defined $t\left(K_{n}\right)$ for $n<m$, then let $t\left(K_{m}\right)$ be a number which appears in the $m$ th column of this matrix, in rows number $k$ for which $t_{k, n}=t\left(K_{n}\right)$ for $n<m$, infinitely many times.

Let $I \subset \mathbb{R}$ be an arbitrary interval. Let $\left(m_{s}\right)_{s}$ be a strictly increasing sequence of those $m \in \mathbb{N}$, for which $K_{m} \subset I$. Take an $\varepsilon>0$ and find an $l \in \mathbb{N}$ such that $\sum_{s>l} r\left(K_{m_{s}}\right)<\varepsilon$. There is a $k \in \mathbb{N}$ such that $t_{k, n}=t\left(K_{n}\right)$ for $n \leq m_{l}$. So by (1),

$$
\begin{aligned}
\left|\sum_{s=1}^{l} t\left(K_{m_{s}}\right) \cdot r\left(K_{m_{s}}\right)\right| & =\left|\sum_{K_{n} \subset I, n \leq m_{l}} t_{k, n} \cdot r\left(K_{n}\right)\right| \\
& \leq 2 \max \left\{r\left(K_{n}\right): K_{n} \subset I, n \leq m_{l}\right\} \\
& \leq 2 \sup \{r(K): K \subset I, K \in \mathscr{K}\} .
\end{aligned}
$$


Hence

$$
\begin{aligned}
\left|\sum_{K \subset I, K \in \mathscr{K}} t(K) \cdot r(K)\right|= & \left|\sum_{s} t\left(K_{m_{s}}\right) \cdot r\left(K_{m_{s}}\right)\right| \\
& \leq\left|\sum_{s=1}^{l} t\left(K_{m_{s}}\right) \cdot r\left(K_{m_{s}}\right)\right|+\sum_{s>l}\left|t\left(K_{m_{s}}\right) \cdot r\left(K_{m_{s}}\right)\right| \\
& <2 \sup \{r(K): K \subset I, K \in \mathscr{K}\}+\varepsilon .
\end{aligned}
$$

Since $\varepsilon$ was an arbitrary positive number, the assertion of the lemma holds.

Proposition 10. Every compact interval belongs to $\mathscr{J}$.

Proof. Since $G_{\xi}=\mathbb{R}$, it is enough to prove by transfinite induction on $\alpha<\Omega$ that every compact interval contained in $G_{\alpha}$ belongs to $\mathscr{J}$.

I. Let $\alpha=0$. Then $G_{\alpha}=\varnothing$ and there is nothing to prove.

II. (a) Assume that the assertion holds for each compact interval contained in $G_{\alpha}$ and $\alpha$ is even. Let $I$ be a compact interval contained in $G_{\alpha+1}$ and $\varepsilon \in(0,1]$. Let $\left\{I_{n}: n\right\}$ be a family (maybe empty) of all components of $I \cap G_{\alpha}$ and $a_{n}=\inf I_{n}, b_{n}=\sup I_{n}$. For each $n$ :

- if $a_{n}, b_{n} \in G_{\alpha}$, then $I \subset G_{\alpha}$ and so by assumption $I \in \mathscr{J}$;

- if $a_{n}, b_{n} \notin G_{\alpha}$, then find a strictly increasing sequence $\left(y_{n, z}\right)_{z \in \mathbf{Z}}$ with limit points $a_{n}$ and $b_{n}$, and such that for each $z \in \mathbb{Z},\left|I_{n, z}\right| \leq$ $\left[\varrho\left(I_{n, z},\left\{a_{n}, b_{n}\right\}\right)\right]^{2}$, where $I_{n, z}=\left[y_{n, z-1}, y_{n, z}\right] \subset G_{\alpha}$; (Note that unlike in the proof of Lemma 6 , we do not require that $y_{n, z} \in T$ for $z \in \mathbb{Z}$.)

- if $a_{n} \in G_{\alpha}$ and $b_{n} \notin G_{\alpha}$, then find a strictly increasing sequence $\left(y_{n, z}\right)_{z \in \mathbb{N}}$ with $y_{n, 1}=a_{n}$ and $\lim _{z \rightarrow \infty} y_{n, z}=b_{n}$, and such that for each $z \in \mathbb{N} \backslash\{1\},\left|I_{n, z}\right| \leq\left[\varrho\left(I_{n, z},\left\{b_{n}\right\}\right)\right]^{2}$, where $I_{n, z}=\left[y_{n, z-1}\right.$, $\left.y_{n, z}\right] \subset G_{\alpha}$;

- similarly if $a_{n} \notin G_{\alpha}$ and $b_{n} \in G_{\alpha}$, then find a strictly increasing sequence $\left(y_{n, z}\right)_{z \in \mathbb{Z} \backslash \mathbb{N}}$ with $y_{n, 0}=b_{n}$ and $\lim _{z \rightarrow-\infty} y_{n, z}=a_{n}$, and such that for each $z \in \mathbb{Z} \backslash \mathbb{N},\left|I_{n, z}\right| \leq\left[\varrho\left(I_{n, z},\left\{a_{n}\right\}\right)\right]^{2}$, where $I_{n, z}=$ $\left[y_{n, z-1}, y_{n, z}\right] \subset G_{\alpha}$.

For each $n$ and $z$, let $\left(f_{n, z, 1}, f_{n, z, 2}\right) \in \mathscr{P}\left(I_{n, z}, \varepsilon / 2^{n+|z|}\right)$. For $j \in\{1,2\}$, define the function $f_{j}: I \rightarrow \mathbb{R}$ by the formula

$$
f_{j}(x)= \begin{cases}f_{n, z, j}(x) & \text { if } x \in I_{n, z}, \\ 1 & \text { if } x \in I \backslash G_{\alpha}\end{cases}
$$

The pair $\left(f_{1}, f_{2}\right)$ obviously satisfies $(\mathrm{a})-(\mathrm{e})$ and $f_{1}, f_{2}$ are derivatives at points of $G_{\alpha} \cap I$. If $x \in I \backslash G_{\alpha}$, then $x \in S$. Let $\tau>0$ be arbitrary and let $k \in \mathbb{N}$ be such that $\tau>\varepsilon / 2^{k}$. Set $\eta=\min \left\{\varrho\left(\{x\}, \bigcup_{n+|z| \leq k} I_{n, z}\right), \tau / 6\right\}$. Let $y \in(0, \eta)$. We may assume that $x+y \in$ int $I_{s, m}$ for some $s$ and $m$ (otherwise we would drop the last two terms in the first line of the estimation below). Then for 
$j \in\{1,2\}$,

$$
\begin{aligned}
\left|\frac{1}{y} \cdot \int_{x}^{x+y} f_{j}-1\right| & \leq \frac{1}{y}\left(\sum_{I_{n, z} \subset(x, x+y]}\left|\int_{I_{n, z}} f_{j}-\right| I_{n, z}||+\int_{I_{s, m}}\left|f_{j}\right|+\left|I_{s, m}\right|\right) \\
& \leq \frac{1}{y}\left(\sum_{I_{n, z} \subset(x, x+y]} \frac{\varepsilon \cdot\left|I_{n, z}\right|}{2^{n+|z|}}+3\left[\varrho\left(\{x\}, I_{s, m}\right)\right]^{2}\right) \\
& \leq \frac{\varepsilon}{2^{k+1}}+3 y<\tau .
\end{aligned}
$$

A similar argument holds for $y \in(-\eta, 0)$. So $f_{1}, f_{2} \in \mathscr{D}(I)$. Finally observe that

$$
\left|\int_{I} f_{1}-\right| I||=\left|\sum_{n} \sum_{z}\left(\int_{I_{n, z}} f_{1}-\left|I_{n, z}\right|\right)\right| \leq \sum_{n} \sum_{z} \frac{\varepsilon \cdot\left|I_{n, z}\right|}{2^{n+|z|}}<\varepsilon \cdot|I|
$$

and

$$
\begin{aligned}
\left|\left\{x \in I: f_{1}(x)=f_{2}(x)=1\right\}\right| & \geq\left|I \backslash G_{\alpha}\right|+\sum_{n} \sum_{z}\left(1-\frac{\varepsilon}{2^{n+|z|}}\right) \cdot\left|S \cap I_{n, z}\right| \\
& \geq\left|I \backslash G_{\alpha}\right|+(1-\varepsilon) \cdot\left|S \cap I \cap G_{\alpha}\right| \\
& \geq(1-\varepsilon) \cdot|S \cap I|,
\end{aligned}
$$

i.e. (f) and (g) are also fulfilled, which proves that $I \in \mathcal{J}$ in this case.

II. (b) Assume that the assertion holds for each compact interval contained in $G_{\alpha}$ and $\alpha$ is odd. First we will prove the following statement:

if $I$ is a bounded interval contained in $G_{\alpha+1}$ and $a=\inf I, b=$ (*) $\quad \sup I \in T$, then there exist derivatives $f_{1}, f_{2}$ defined on cl $I$ which satisfy (a)-(e) and such that $f_{1}=f_{2}=0$ on $\operatorname{cl} I \backslash G_{\alpha}$.

Let $\left\{I_{n}: n\right\}$ be a family (maybe empty) of all components of $I \cap G_{\alpha}$, and let $a_{n}, b_{n}$ be endpoints of $I_{n}$. For each $n$, use Lemma 6 to find a strictly increasing sequence $\left(y_{n, z}\right)_{z \in \mathbb{Z}}$ of elements of $T \cap I_{n}$ with limit points $a_{n}$ and $b_{n}$, and such that for each $z \in \mathbb{Z},\left|I_{n, z}\right| \leq \varphi\left(I_{n, z}\right)+\left[\varrho\left(I_{n, z},\left\{a_{n}, b_{n}\right\}\right)\right]^{2}$, where $I_{n, z}=\left[y_{n, z-1}, y_{n, z}\right] \subset G_{\alpha}$. For each $n$ and each $z \in \mathbb{Z}$, let $\left(f_{n, z, 1}, f_{n, z, 2}\right) \in$ $\mathscr{P}\left(I_{n, z}, 1\right)$ and define $r\left(I_{n, z}\right)=\int_{I_{n, z}} f_{n, z, 1}$. Use Lemma 9 for the family $\mathscr{K}=\left\{I_{n, z}: n, z \in \mathbb{Z}\right\}$ and the function $r$, and find a function $t: \mathscr{K} \rightarrow\{-1,1\}$ such that for every interval $J \subset \mathbb{R}$,

$$
\left|\sum_{I_{n, z} \subset J} t\left(I_{n, z}\right) \cdot r\left(I_{n, z}\right)\right| \leq 2 \sup \left\{r\left(I_{n, z}\right): I_{n, z} \subset J\right\} .
$$

For $j \in\{1,2\}$, define the function $f_{j}: \operatorname{cl} I \rightarrow \mathbb{R}$ as follows:

$$
f_{j}(x)= \begin{cases}t\left(I_{n, z}\right) \cdot f_{n, z, j}(x) & \text { if } x \in I_{n, z}, \\ 0 & \text { if } \ddot{z} \in \operatorname{cl} I \backslash G_{\alpha} .\end{cases}
$$


The pair $\left(f_{1}, f_{2}\right)$ obviously satisfies (a)-(e) and $f_{1}, f_{2}$ are derivatives at points of $G_{\alpha} \cap I \backslash\{a, b\}$. If $x \in I \backslash G_{\alpha}$ or $x \in\{a, b\}$, then $x \in T$. Let $y>0$. We may assume that $x+y \in \operatorname{int} I_{s, m}$ for some $s$ and some $m \in \mathbb{Z}$ (otherwise we would drop the last term in the first line of the estimation below). Then for $j \in\{1,2\}$,

$$
\begin{aligned}
\left|\frac{1}{y} \int_{x}^{x+y} f_{j}\right| \leq & \frac{1}{y}\left(\left|\sum_{I_{n, z} \subset(x, x+y]} \int_{I_{n, z}} f_{j}\right|+\int_{I_{s, m}}\left|f_{j}\right|\right) \\
\leq & \frac{1}{y}\left(\left|\sum_{I_{n, z} \subset(x, x+y]} t\left(I_{n, z}\right) \cdot r\left(I_{n, z}\right)\right|+2\left|I_{s, m}\right|\right) \\
\leq & \frac{2 \sup \left\{r\left(I_{n, z}\right): I_{n, z} \subset(x, x+y]\right\}}{y}+\frac{2 \cdot \varphi\left(I_{s, m}\right)(1+y)}{y+y^{2}}+2 y \\
\leq & \frac{4 \sup \left\{\left|I_{n, z}\right|: I_{n, z} \subset(x, x+y]\right\}}{y} \\
& +\frac{2 \cdot \varphi\left(x, x+y+\left|I_{s, m}\right|\right) \cdot(1+y)}{y+\left|I_{s, m}\right|-\varphi\left(I_{s, m}\right)}+2 y \\
\leq & \frac{4 \cdot \varphi(x, x+y)}{y}+\frac{2 \cdot \varphi\left(x, x+y+\left|I_{s, m}\right|\right)}{y+\left|I_{s, m}\right|} \\
& \cdot \frac{1+y}{1-\frac{\varphi\left(x, x+y+\left|I_{s, m}\right|\right)}{y+\left|I_{s, m}\right|}+6 y \stackrel{y \rightarrow 0}{\longrightarrow} 0 .}
\end{aligned}
$$

(We used the fact that $T$ is nonporous at $x$.) Similar argument holds for $y<0$, so $f_{1}, f_{2} \in \mathscr{D}(\mathrm{cl} I)$, which completes the proof of $(*)$.

Now let $I$ be any compact interval contained in $G_{\alpha+1}$ and $\varepsilon \in(0,1]$.

- Use Lemma 3 to find nonoverlapping compact intervals $J_{1}, \ldots, J_{n}$ contained in $I \cap G_{\alpha}$ such that $\operatorname{fr}\left(I \cap G_{\alpha} \backslash \bigcup_{i=1}^{n} J_{i}\right) \subset T,\left|I \cap G_{\alpha} \backslash \bigcup_{i=1}^{n} J_{i}\right| \leq$ $\frac{\varepsilon^{\bullet}\left|I \cap G_{\alpha}\right|}{6}$ and $\left|S \cap I \backslash \bigcup_{i=1}^{n} J_{i}\right| \leq \frac{\varepsilon^{*}|S \cap I|}{2}$. For $i \in\{1, \ldots, n\}$, let $\left(g_{i, 1}\right.$, $\left.g_{i, 2}\right) \in \mathscr{P}\left(J_{i}, \varepsilon / 2\right)$.

- Let $I_{1}, \ldots, I_{m}$ be components of $I \backslash \bigcup_{i=1}^{n} J_{i}$. For $k \in\{1, \ldots, m\}$, use $(*)$ with $I=I_{k}$ and find derivatives $h_{k, 1}, h_{k, 2}$ defined on $\mathrm{cl} I_{k}$ which satisfy (a)-(e).

- Use Lemma 7 with $D=I \backslash G_{\alpha}$ and $\varepsilon_{1}=\varepsilon_{2}=1-\varepsilon$, and find approximately continuous functions $f_{0,1}, f_{0,2}$ satisfying conditions (1)-(4) of that lemma.

- For $j \in\{1,2\}$, define the function $f_{j}: I \rightarrow \mathbb{R}$ as follows:

$$
f_{j}(x)=f_{0, j}(x)+ \begin{cases}g_{i, j}(x) & \text { if } x \in J_{i}, \quad i \in\{1, \ldots, n\} \\ h_{k, j}(x) & \text { if } x \in I_{k}, \quad k \in\{1, \ldots, m\}\end{cases}
$$

Then obviously $f_{1}$ and $f_{2}$ are derivatives which satisfy (a)-(e). (Note that 
$h_{k, j}=0$ at the endpoints of $I_{k}$ and on $I_{k} \backslash G_{\alpha}, k \in\{1, \ldots, m\}$.) Moreover,

$$
\begin{aligned}
\left|\int_{I} f_{1}-\right| I|| \leq & \left|\int_{\bigcup_{i=1}^{n} J_{i}} f_{1}-\right| \bigcup_{i=1}^{n} J_{i}||+\left|\int_{I \cap G_{\alpha} \backslash \bigcup_{i=1}^{n} J_{i}} f_{1}-\right| I \cap G_{\alpha} \backslash \bigcup_{i=1}^{n} J_{i}|| \\
& +\left|\int_{I \backslash G_{\alpha}} f_{1}-\right| I \backslash G_{\alpha}|| \\
\leq & \frac{\varepsilon}{2} \cdot\left|\bigcup_{i=1}^{n} J_{i}\right|+3 \cdot\left|I \cap G_{\alpha} \backslash \bigcup_{i=1}^{n} J_{i}\right|+\varepsilon \cdot\left|I \backslash G_{\alpha}\right| \\
\leq & \frac{\varepsilon}{2} \cdot\left|\bigcup_{i=1}^{n} J_{i}\right|+\frac{\varepsilon}{2} \cdot\left|I \cap G_{\alpha}\right|+\varepsilon \cdot\left|I \backslash G_{\alpha}\right| \leq \varepsilon \cdot|I|,
\end{aligned}
$$

and

$$
\begin{aligned}
\left|\left\{x \in I: f_{1}(x)=f_{2}(x)=1\right\}\right| & \geq \sum_{i=1}^{n}\left|\left\{x \in J_{i}: f_{1}(x)=f_{2}(x)=1\right\}\right| \\
& \geq \sum_{i=1}^{n}\left(1-\frac{\varepsilon}{2}\right) \cdot\left|S \cap J_{i}\right| \\
& \geq\left(1-\frac{\varepsilon}{2}\right)\left(1-\frac{\varepsilon}{2}\right) \cdot|S \cap I| \geq(1-\varepsilon) \cdot|S \cap I|,
\end{aligned}
$$

so (f) and (g) are also satisfied, which proves that $I \in \mathscr{J}$ in this case.

III. Assume that $\alpha$ is a limit ordinal and that every compact interval in $G_{\beta}$ with some $\beta<\alpha$ belongs to $\mathcal{J}$. Let $I$ be an arbitrary compact interval contained in $G_{\alpha}$. Then by the compactness of $I$ we get $I \subset G_{\beta_{1}} \cup \cdots \cup G_{\beta_{n}}=G_{\gamma}$, where $\gamma=\max \left\{\beta_{i}: i \in\{1, \ldots, n\}\right\}<\alpha$. Hence by assumption $I \in \mathscr{J}^{\prime}$.

This completes the proof.

Theorem 11. Assume that $S \subset \mathbb{R}$ is ambiguous and $T=\mathbb{R} \backslash S$ is nonporous. Then there exist derivatives $f, g \in \mathscr{D}$ such that

(i) $f \cdot g=0$ on $T$,

(ii) $f=g$ and $|f|=1$ on $S$,

(iii) $|f|<2,|g|<2$.

Proof. Let $I_{z}=[z-1, z]$ for $z \in \mathbb{Z}$. By Proposition 10, $I_{z} \in \mathcal{J}$. Let, e.g., $\left(f_{z}, g_{z}\right) \in \mathscr{P}\left(I_{z}, 1\right)(z \in \mathbb{Z})$. Define

$$
\begin{array}{ll}
f(x)=f_{z}(x) & \text { if } x \in I_{z}, z \in \mathbb{Z}, \\
g(x)=g_{z}(z) & \text { if } x \in I_{z}, z \in \mathbb{Z} .
\end{array}
$$

It is easy to verify that $f$ and $g$ satisfy our requirements.

In $1990 \mathrm{~J}$. Marík proved the following theorem.

Theorem 12. Let $S \subset \mathbb{R}, T=\mathbb{R} \backslash S$. Then the following three conditions are equivalent:

(1) There is a natural number $m$ and derivatives $f_{1}, \ldots, f_{m}$ such that

$$
f_{1} \cdots f_{m}=\chi_{s}
$$

(2) $S$ is ambiguous and $T$ is nonporous. 
(3) There are $f, g \in \mathscr{D}$ such that $f=g=1$ on $S$ and $f g=0$ on $T$ [4, Theorem 18].

Hence and by Theorem 11 we get the following corollary.

Corollary 13. Let $S \subset \mathbb{R}, T=\mathbb{R} \backslash S$. Then the following four conditions are equivalent:

(1) There is a natural number $m$ and derivatives $f_{1}, \ldots, f_{m}$ such that

$$
f_{1} \cdots f_{m}=\chi_{S}
$$

(2) $S$ is ambiguous and $T$ is nonporous.

(3) There are $f, g \in \mathscr{D}$ such that $f=g$ and $|f|=1$ on $S, f g=0$ on $T$ and $|f|<2,|g|<2$ on $\mathbb{R}$.

(4) There is a natural number $m$ and bounded derivatives $f_{1}, \ldots, f_{m}$ such that

$$
f_{1} \cdots f_{m}=\chi_{S}
$$

\section{ACKNOWLEDGMENT}

I would like to thank Professor Konjagin for Lemmas 8 and 9, which helped me to shorten the proof of Proposition 10 and to improve the assertion of Theorem 11.

\section{REFERENCES}

1. A. M. Bruckner, Differentiation of real functions, Lecture Notes in Math., vol. 659, SpringerVerlag, Berlin and New York, 1978.

2. C. Goffman, C. J. Neugebauer, and T. Nishiura, Density topology and approximate continuity, Duke Math. J. 28 (1961), 497-506.

3. C. Kuratowski, Topologie, Vol. I, PWN, Warszawa, 1958.

4. J. Mařik, Characteristic functions and products of derivatives, Real Anal. Exchange 16 (1990-1991), 245-254.

5. G. Petruska and M. Laczkovich, Baire one functions, approximately continuous functions and derivatives, Acta Math. Acad. Sci. Hungar. 25 (1974), 189-212.

6. Z. Zahorski, Sur la première dérivée, Trans. Amer. Math. Soc. 69 (1950), 1-54.

Department of Mathematics, Pedagogical University, ul. Chodkiewicza 30, 85-064 Bydgoszcz, Poland

E-mail address: wspb050pltumk11.bitnet 